\title{
The Activity of Matrix Metalloproteinases (MMPs) and Tissue Inhibitors of Metalloproteinases (TIMPs) in Mammary Tumors of Dogs and Rats
}

\author{
Kouji KAWAI ${ }^{1)}$, Koji UETSUKA ${ }^{1)}$, Kunio DOI ${ }^{1)}$ and Hiroyuki NAKAYAMA ${ }^{1) *}$ \\ ${ }^{1)}$ Department of Veterinary Pathology, Graduate School of Agricultural and Life Sciences, The University of Tokyo, 1-1-1 Yayoi, Bunkyo- \\ ku, Tokyo 113-8657, Japan
}

(Received 17 June 2005/Accepted 16 September 2005)

\begin{abstract}
We conducted zymography for detecting the activity of matrix metalloproteinases (MMPs) and reverse zymography for the activity of tissue inhibitors of metalloproteinases (TIMPs) in canine spontaneous and rat 7, 12-dimethylbenz(a)anthracene (DMBA)induced mammary tumor tissues. The activities of MMPs of canine mammary tumors were quite higher than those of the rat chemically induced tumors. The activities of MMPs were significantly higher in malignant tissues than in benign ones of canine tumors, whereas the activity of only MMP-2 was higher in both benign and malignant rat tumors compared to normal tissues. There were no differences of MMPs activities between benign and malignant rat tumors. The results of reverse zymography indicated that the activities of TIMP$1,-2$ and -3 were strikingly higher in rat tumors than in canine tumors. The activities were higher in malignant tissues than in benign ones of dogs, and higher in tumor tissues than in normal mammary tissues of rats. The results of film in situ zymography for tissue localization of gelatinolytic activity showed that the digested area was more extended in malignant tumors than in benign ones of dogs. However, the area was similarly extended in both benign and malignant rat tumors. These results may indicate that the canine spontaneous malignant mammary tumors possess more aggressive nature than the rat chemically induced counterpart, resulting from the high level of MMPs and low level of TIMPs activities of the tumor tissues.

KEY WORDS: canine, mammary tumor, MMP, rat, TIMP.
\end{abstract}

J. Vet. Med. Sci. 68(2): 105-111, 2006

Malignant tumors often metastasize to other organs such as lymph nodes and lungs, which often influences the prognosis of the patients. Destruction of the basement membrane is said to be one of the most important steps for tumor metastasis [11]. Matrix metalloproteinases (MMPs) belong to a family of zinc-dependent proteases and are capable of degrading basement membrane as well as extracellular matrix surrounding cells. It is widely known that MMPs are important for tumor invasion and metastasis [25]. Of MMPs, MMP-2 and MMP-9 have gelatinase activity, and are also known as type IV collagenases. The basement membrane consisting of type IV collagen functions as a barrier against carcinoma invasion and metastasis. A part of MMPs activities are controlled by tissue inhibitors of metalloproteinases (TIMPs). TIMPs are known not only to inhibit MMPs activity but to play a role for activation of some MMPs [10]. The total enzymatic activities of MMPs are determined by the activity of both latent and active forms of MMPs, and by the inhibition of the activity by TIMPs as well [15]. The unbalanced activities of MMPs and TIMPs are involved in tumor progression [12]. Tissue activities of MMP-2 and -9 are reported to correlate well with malignancy and prognosis in human mammary tumors $[2,6,13,20,21,28]$.

Mammary tumors are the most common tumor in dogs as well as in human. Malignant cases may occupy approximately a half of canine mammary tumors [1]. Activities of

\footnotetext{
* Correspondence to: Nakayama, H., Department of Veterinary Pathology, Graduate School of Agricultural and Life Sciences, The University of Tokyo, 1-1-1 Yayoi, Bunkyo-ku, Tokyo 1138657, Japan.
}

MMP-2 and -9 correlate well with malignancy and prognosis in canine mammary tumors $[13,28]$ as in human ones. However, there are no reports regarding TIMPs activities in canine mammary tumors. In the present study, we examined both MMPs and TIMPs activities in spontaneous canine mammary tumors using zymography and reverse zymography, and also performed film in situ zymography (FIZ) method to detect gelatinolytic activity on a tissue section. FIZ method was applied to several human cancers, and reported to correlate with their malignancy [7, 8, 24, 29]. Localization of MMP-2 expression in canine mammary tumors is recently reported [19], but that of the enzymatic activity in situ is still unknown.

We also examined the MMPs and TIMPs activities of chemically induced mammary tumors in Sprage-Dawley (SD) rats by administering 7, 12-dimethylbenz(a)anthracene (DMBA). Tumor invasion and metastasis are very rare in the rat model though they are common in canine malignant tumors [22]. Therefore, some different mechanisms may determine the tumor progression between spontaneous canine tumors and chemically induced rat tumors. The balance of MMPs/TIMPs activities is compared in the tumors of the species.

\section{MATERIALS AND METHODS}

Canine mammary tumors: All the samples of canine mammary tumors used in the present study were collected from biopsy cases at the Department of Veterinary Pathology, the University of Tokyo. A half of each sample was fixed in $10 \%$ neutral buffered formalin, and paraffin sec- 
tions of $4 \mu \mathrm{m}$ thick were stained with hematoxylin and eosin (HE). The other half of the sample was stored at $\quad-80^{\circ} \mathrm{C}$ and used for making frozen sections and for performing protein and RNA extraction. According to the histopathological classification proposed by WHO [16], 10 adenocarcinomas and 10 adenomas were chosen for zymography and reverse zymography. FIZ was performed using 9 samples of the mammary tumors.

DMBA-induced mammary tumors of SD rats: Female SD rats were purchased from Japan SLC (Hamamatsu, Japan). Animals were kept in isolator cages (Niki Shoji, Tokyo, Japan) under a controlled condition $\left(23 \pm 2{ }^{\circ} \mathrm{C}\right.$ with $55 \pm 5 \%$ humidity and a $14 \mathrm{hr}$ light/10 hr dark cycle) and fed commercial diet pellets (MF; Oriental Yeast, Tokyo, Japan) and water ad libitum during experiments. The tumor induction was conducted according to the report by Huggins et al. [5]. The animals received a single intragastric administration of DMBA $(20 \mathrm{mg} / \mathrm{rat})$ dissolved in sesame oil at 50 days of age. The number and location of induced tumors were recorded each week until the termination of the experiment (13 weeks). After the animals were humanly sacrificed with ethyl ether, mammary tumors were excised and weighed. A half of the tissue was fixed in 10\% neutral buffered formalin, and paraffin sections of $4 \mu \mathrm{m}$ thick were stained with HE for histopathological examination. The other half was stored at $-80^{\circ} \mathrm{C}$ and used for making frozen sections and performing protein extraction.

Protein extraction: Frozen tumor samples were minced and homogenized in three volumes of extraction buffer $(1 \%$ NP-40, 50 mM Tris-HCl, pH 7.4, 0.25\% Na-deoxycholate, $150 \mathrm{mM} \mathrm{NaCl}, 1 \mathrm{mM}$ phenylmethane sulfonyl fluoride, 2 $\mu \mathrm{g} / \mathrm{m} l$ aprotinin, and $2 \mu \mathrm{g} / \mathrm{m} l$ leupeptin). The homogenate was centrifuged at $10,500 \times \mathrm{g}$ for $20 \mathrm{~min}$ and the supernatant was used for zymography and reverse zymography. The protein concentration of the supernatant was measured by the Lowry's method using the DC protein assay kit (BioRad, Hercules, CA, U.S.A.).

Zymography and reverse zymography: Gelatin zymography was performed to detect MMP-2 and MMP-9 activities in the extracted samples from mammary gland tumors using the methods described previously [9] with a minor modification. Proteins in a sample were separated by sodium dodecyl sulphate (SDS)-polyacrylamide gel electrophoresis (SDS-PAGE) using 10\% gels containing $1 \mathrm{mg} / \mathrm{m} l$ gelatin. MMP-2 and MMP-9 protein standards (Oncogene, San Diego, CA, U.S.A.) were used as positive controls. Samples were electrophoresed at $20 \mathrm{~mA}$ for $70 \mathrm{~min}$. The gels were washed in a washing buffer $(50 \mathrm{mM}$ Tris, $0.2 \mathrm{M} \mathrm{NaCl}$, and $2.5 \%$ Triton $\mathrm{X}-100)$ for $1 \mathrm{hr}$, and then incubated in a digestion buffer ( $50 \mathrm{mM}$ Tris, $0.2 \mathrm{M} \mathrm{NaCl}, 0.02 \%$ Brij-35, and 5 $\mathrm{mM} \mathrm{CaCl} 2$ ) at $37^{\circ} \mathrm{C}$ for $20 \mathrm{hr}$. The gels were stained with 2.5\% Coomassie Blue R-250 in 50\% methanol and 10\% acetic acid for $20 \mathrm{~min}$, and destained in $30 \%$ methanol and $10 \%$ acetic acid solution, until active bands become clear. Reverse zymography was performed by almost the same procedure, except that the concentration of gel was $15 \%$ without SDS, and that the gel contained $0.15 \mu \mathrm{g} / \mathrm{m} l$ recombinant human (rh) Pro-MMP-2 [18]. Control proteins (TIMP-1 and -2 from human fibroblasts, rhTIMP-3 and rhTIMP-4) for reverse zymography were purchased from Oncogene (Cambridge, MA, U.S.A.).

Film in situ zymography: Detection of in situ gelatinolytic activity was performed using FIZ method (FIZ-GN). Films which are coated with a $7 \mu \mathrm{m}$-thick layer of cross-linked gelatin (FIZ-GN film), and those coated with 1,10-phenanthorolin (an MMP inhibitor) as negative controls (FIZ-GI film), were commercially provided by Wako Pure Chemical, Tokyo, Japan. Six- $\mu$ m frozen tissue sections were mounted with the films. The film-mounted sections were then incubated at $37^{\circ} \mathrm{C}$ for $7.5 \mathrm{hr}$ in a moisture chamber.

Table 1. Canine mammary gland tumors used in the study

\begin{tabular}{|c|c|c|c|c|c|}
\hline & Case & Breed & Sex & Age & Diagnosis \\
\hline \multirow{10}{*}{ Carcinoma } & 1 & Chihuahua & Female & $9 y 9 m$ & Tubulopapillary carcinoma \\
\hline & 2 & Golden retriever & Female & $11 \mathrm{y} 0 \mathrm{~m}$ & Tubulopapillary carcinoma \\
\hline & 3 & Shih Tzu & Female & $15 \mathrm{y} 0 \mathrm{~m}$ & Tubulopapillary carcinoma \\
\hline & 4 & Golden retriever & Female & $9 \mathrm{y} 0 \mathrm{~m}$ & Solid carcinoma \\
\hline & 5 & Mix & Spayed female & $11 \mathrm{y} 0 \mathrm{~m}$ & Solid carcinoma \\
\hline & 6 & Beagle & Spayed female & $11 \mathrm{y} 5 \mathrm{~m}$ & Solid carcinoma \\
\hline & 7 & Mix & Spayed female & $12 \mathrm{y} 0 \mathrm{~m}$ & Solid carcinoma \\
\hline & 8 & Mix & Female & $12 \mathrm{y} 10 \mathrm{~m}$ & Solid carcinoma \\
\hline & 9 & Shiba & Spayed female & $5 \mathrm{y} 0 \mathrm{~m}$ & Complex carcinoma \\
\hline & 10 & Golden retriever & Female & $8 y 5 \mathrm{~m}$ & Anaplastic carcinoma \\
\hline \multirow{10}{*}{ Adenoma } & 11 & Pomeranian & Female & $5 y 3 m$ & Simple adenoma \\
\hline & 12 & Yorkshire terrier & Female & $10 \mathrm{y} 11 \mathrm{~m}$ & Simple adenoma \\
\hline & 13 & Maltese & Female & $11 \mathrm{y} 1 \mathrm{~m}$ & Simple adenoma \\
\hline & 14 & Shih Tzu & Female & $12 \mathrm{y} 0 \mathrm{~m}$ & Simple adenoma \\
\hline & 15 & Mix & Spayed female & $15 \mathrm{y} 10 \mathrm{~m}$ & Simple adenoma \\
\hline & 16 & Miniature Duchshund & Female & $5 y 8 m$ & Complex adenoma \\
\hline & 17 & American cocker spaniel & Female & $5 \mathrm{y} 9 \mathrm{~m}$ & Complex adenoma \\
\hline & 18 & Miniature Duchshund & Female & $6 y 0 \mathrm{~m}$ & Complex adenoma \\
\hline & 19 & Yorkshire terrier & Female & $10 \mathrm{y} 3 \mathrm{~m}$ & Complex adenoma \\
\hline & 20 & Shih Tzu & Female & $13 \mathrm{y} 4 \mathrm{~m}$ & Complex adenoma \\
\hline
\end{tabular}


a)

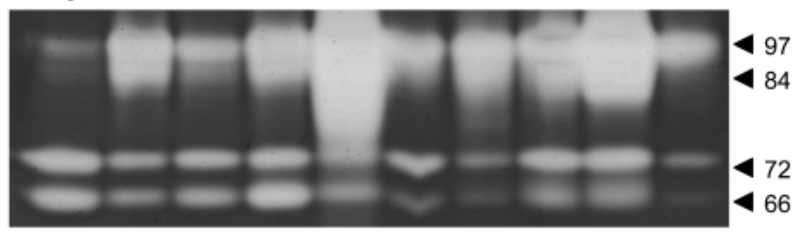

b)

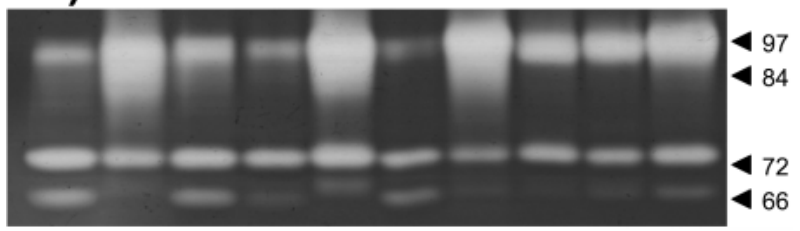

C)

$\square$ Carcinoma

$\square$ Adenoma

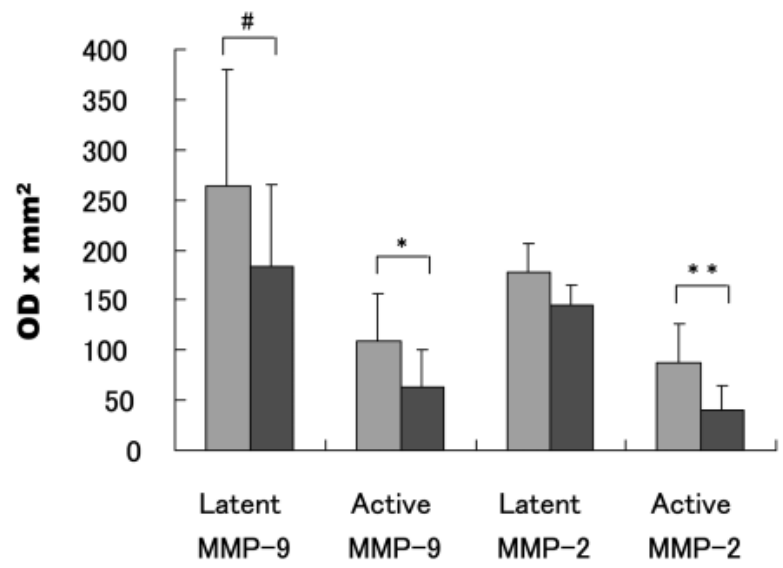

Fig. 1. Activities of MMP-2 and MMP-9 in canine mammary tumors. Gelatin zymography of mammary carcinoma (a) and adenoma (b). Bands of 97, 84, 72 and $66 \mathrm{kDa}$ indicate latent MMP-9, active MMP-9, latent MMP-2 and active MMP-2, respectively. Each lane represents individual case. From left to right; Cases 1 to 10 in (a) and Cases 11 to 20 in (b). Panel (c) is a quantitative representation of the activities. *: $P<0.05, * *$ : $P<0.01$ (Student's $t$-test), and \#: $P<0.05$ (Welch's $t$-test).

After incubation, the films were air-dried for $30 \mathrm{~min}$ and stained with Biebrich-Scaret Red (Wako Pure Chemical) for 4 min.

Statistical analyses: Statistical significance was determined by Student's $t$-test or Welch's $t$-test for the canine data, following the determination of homogeneity of variance by $F$-test. One-way ANOVA was applied for rats' data. Pearson's correlation test was used to determinate the significance for correlation. A significance level of $P<0.01$ or $P<0.05$ was considered acceptable. a)

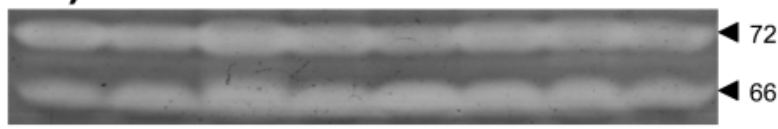

b)

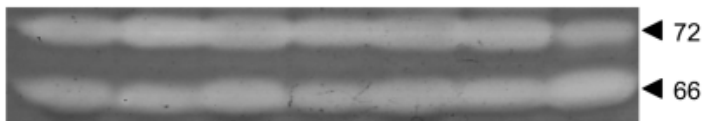

c)

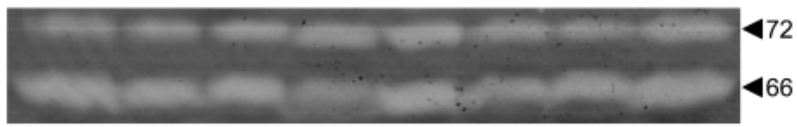

d)

$\square$ Carcinoma

$\square$ Adenoma

$\square$ Normal

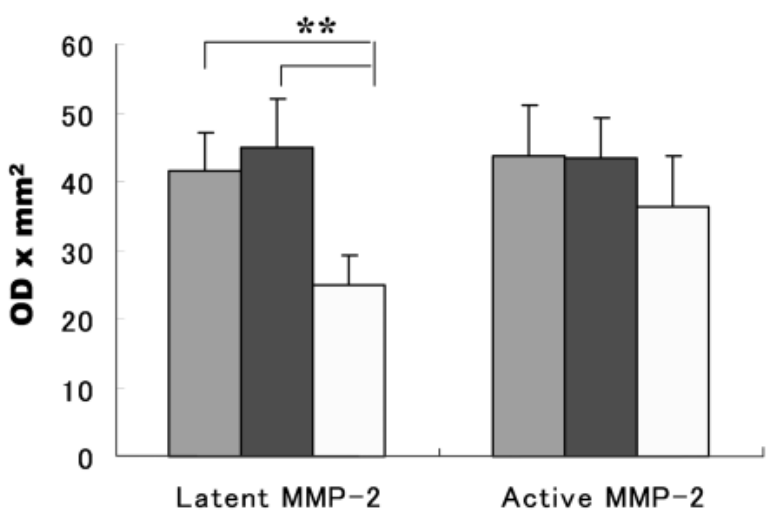

Fig. 2. Activities of MMP-2 in chemically induced mammary tumors in rats. Gelatin zymography of mammary carcinoma (a), adenoma (b) and normal mammary tissue (c). Bands of 72 and $66 \mathrm{kDa}$ indicate latent MMP-2 and active MMP-2, respectively. Each lane represents individual case randomly selected. Panel (d) is a quantitative representation of the activities. ${ }^{* *}: P<0.01$ (One-way ANOVA).

\section{RESULTS}

Canine mammary tumors: Details and histopathological diagnoses of 20 canine mammary tumor cases examined in the study are listed in Table 1 . The diagnoses were 10 malignant tumors including 5 solid carcinomas, 3 tubulopapillary carcinomas, 1 anaplastic carcinoma and 1 complex carcinoma, and 10 benign tumors including 5 simple adenomas and 5 complex adenomas.

DMBA-induced mammary tumors in SD rats: Mammary tumors were detected by palpation at 8 weeks or later after DMBA administration. Tumors were observed in 42 of 45 rats which survived until the end of the experiment. The tumors were histologically diagnosed according to the pre- 
a)

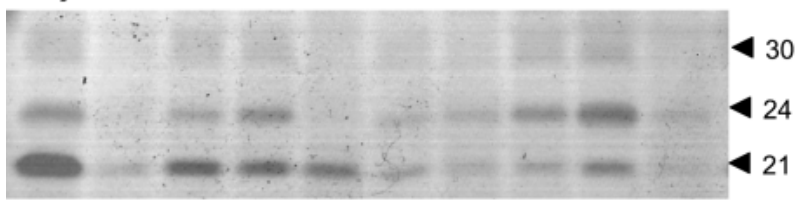

b)

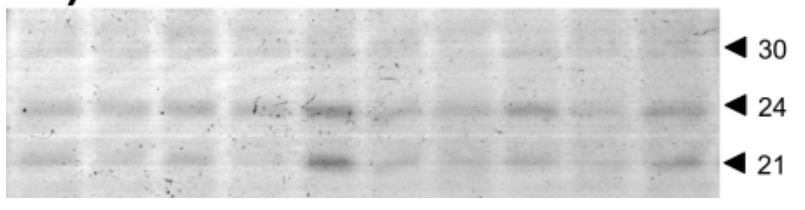

c)

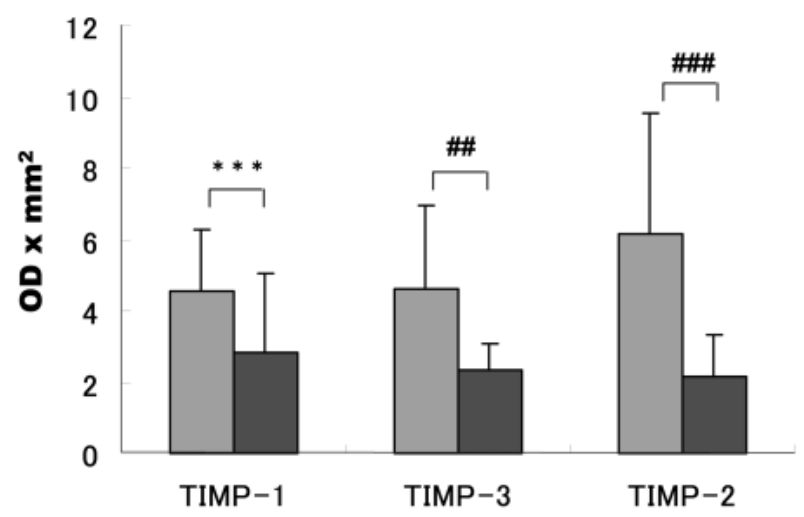

Fig. 3. Activities of TIMP-1, -2 and -3 in canine mammary tumors. Gelatin reverse zymography of mammary carcinoma (a) and adenoma (b). Bands of 30, 24 and $21 \mathrm{kDa}$ indicate TIMP-1, 3 and -2 , respectively. Each lane represents individual case. From left to right; Cases 1 to 10 in (a) and Cases 11 to 20 in (b). Panel (c) is a quantitative representation of the activities. ***: $P<0.001$ (Student's $t$-test), \#\#: $P<0.01$, and \#\#\#: $P<0.001$ (Welch's $t$-test).

vious report [23]. Of 211 mammary gland tumors, 202 were malignant including 42 papillary carcinomas, 99 cribriform carcinomas, 3 comedo carcinomas, and 58 tubular carcinoma, whereas only 9 were benign including 7 lactating adenomas, and 2 tubular adenomas. A precancerous lesion of ductal carcinoma in situ was observed in one rat treated with DMBA and without any other mammary tumors. Normal mammary glands were scattered in the ventral subcutaneous fat tissue.

Zymography: The zymographic band patterns of protein extracts from canine mammary carcinomas and adenomas are shown in Fig. 1. Bands of latent MMP-9 (97 kDa) and MMP-2 (72 kDa) were clearly detected in all the benign and malignant tumor samples examined, whereas active forms of MMP-9 (84 kDa) and MMP-2 (66 kDa) were less expressed in some samples. Enzymatic activities of latent
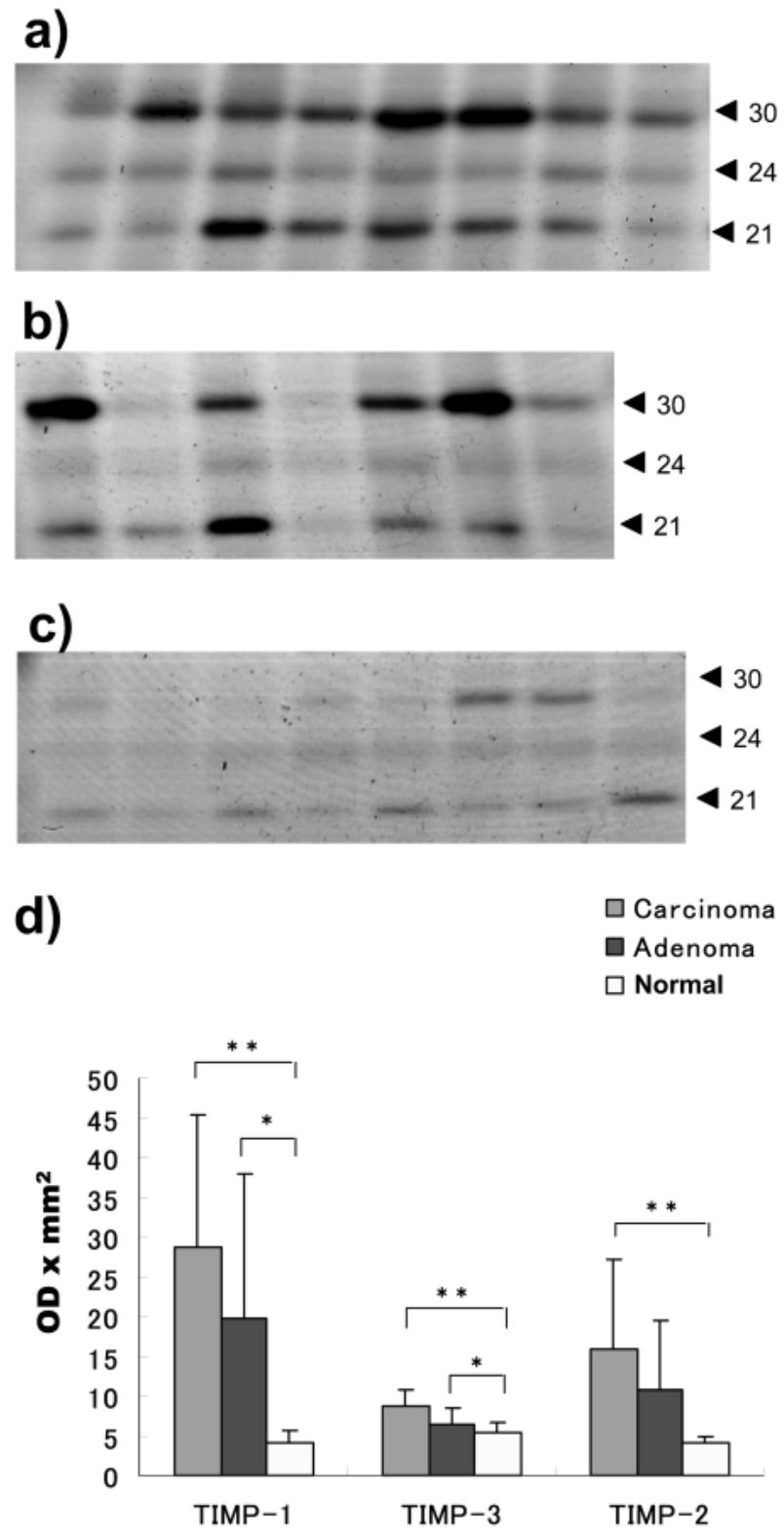

Fig. 4. Activities of TIMP-1, -2 , and -3 in chemically induced mammary tumors of rats. Gelatin reverse zymography of mammary carcinoma (a), adenoma (b) and normal mammary tissue (c). Bands of 30, 24 and $21 \mathrm{kDa}$ are TIMP-1, -3 and -2 , respectively. Each lane represents individual case randomly selected. Panel (d) is a quantitative representation of the activities. *: $P<0.05$, and $* *: P<0.01$ (One-way ANOVA).

MMP-9 as well as active forms of MMP-2 and MMP-9 in carcinomas were significantly higher than those in adenomas, which is consistent with previous reports. The zymographic band patterns of mammary tumors and normal mammary glands from rats are shown in Fig. 2. Bands of latent $(72 \mathrm{kDa})$ and active $(66 \mathrm{kDa})$ forms of MMP-2 were 

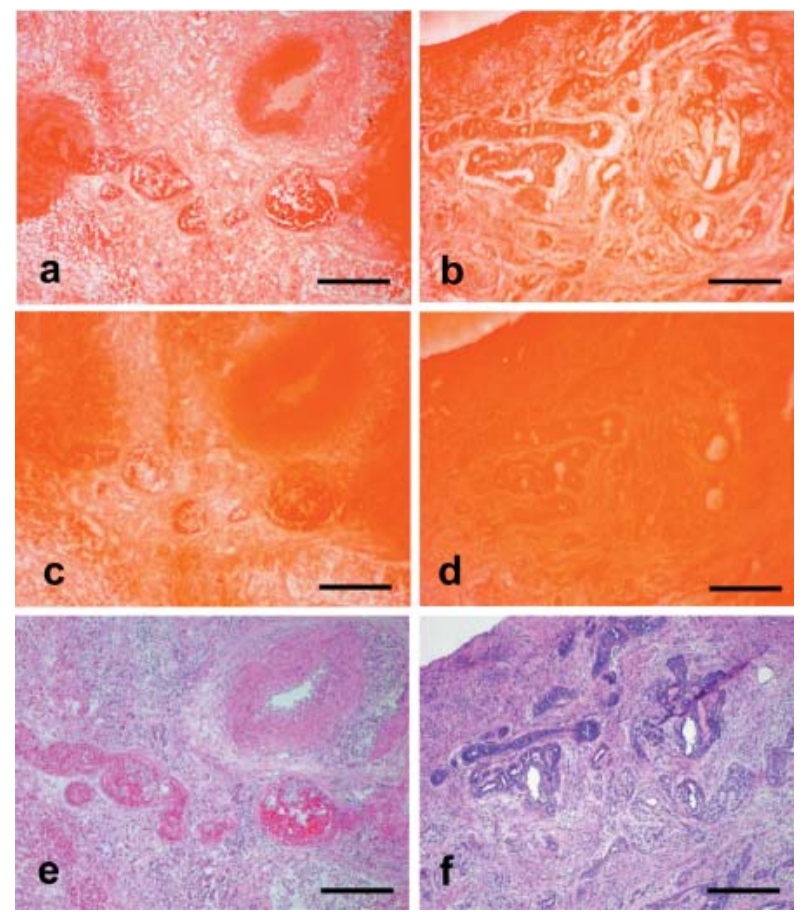

Fig. 5. In situ gelatinolytic activities in the canine mammary tumor by film in situ zymography (FIZ). Mammary carcinoma (a, c and e) and adenoma (b, d and f). Sections coated with cross-linked gelatin alone (FIZ-GN) (a and b), and those coated with cross-linked gelatin including an MMP inhibitor, 1,10phenanthorolin (FIZ-GI) (c and d). HE stain sections of the same areas (e and f). A strong gelatinolytic activity was observed in carcinoma samples but not in adenoma. The gelatinolytic activity was weakened by using FIZ-GI films. Bar $=500 \mu \mathrm{m}$ (a-f).

detected in all the samples examined, although the densities of the bands were strikingly lower than those of canine samples. MMP-9 activity was not clear in rat cases (data not shown). Gelatinolytic activity of latent MMP-2 in tumor samples was higher than that in normal mammary tissues, while there were no differences between carcinoma and adenoma. Activity of active form of MMP-2 seemed to be at the same level among carcinoma, adenoma, and normal mammary tissues.

Reverse zymography: The reverse zymographic band patterns of protein extracts from canine mammary carcinoma and adenoma are shown in Fig. 3. Activities of TIMP-1, -3 and -2 were detected as bands of $30 \mathrm{kDa}, 24 \mathrm{kDa}$, and 21 $\mathrm{kDa}$, respectively, whereas those were extremely low when compared with those of rat tumors. Activities of all the TIMPs examined in canine carcinomas were significantly higher than those in adenomas. The same results were obtained in the experiment using rat tissues (Fig. 4) except for higher activity levels of the enzymes. The highest TIMPs activity was observed in carcinomas, followed by adenomas and normal mammary tissues. TIMP-4 activity was not detectable in the present study.

Film in situ zymography: The FIZ-GN film method
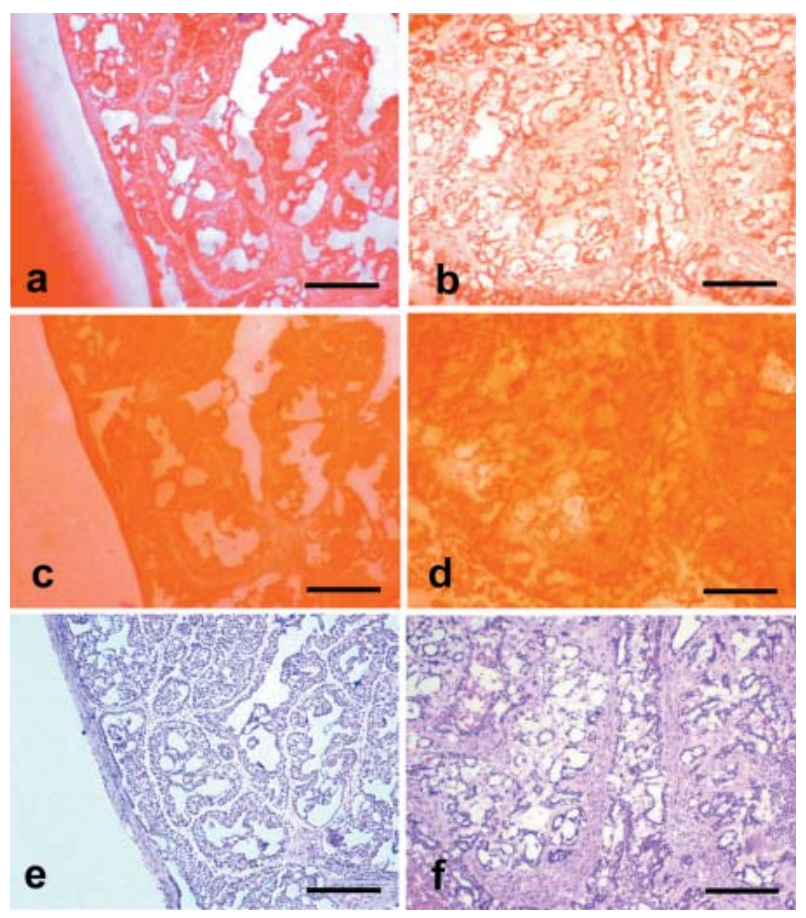

Fig. 6. In situ gelatinolytic activities in the rat mammary tumor by FIZ. Mammary carcinoma (a, c and e) and adenoma (b, d and f). Sections coated with cross-linked gelatin alone (FIZ-GN) (a and $\mathrm{b}$ ), and those coated with cross-linked gelatin including an MMP inhibitor, 1,10-phenanthorolin (FIZ-GI) (c and d). HE stain sections of the same areas (e and $\mathrm{f}$ ). A strong gelatinolytic activity was observed both in carcinoma and adenoma samples. The gelatinolytic activity was weakened by using FIZ-GI films. Bar $=500 \mu \mathrm{m}(\mathrm{a}-\mathrm{f})$.

revealed in situ gelatinolytic activity in mammary tumor samples from dogs and rats. In canine cases, a strong gelatinolytic activity was observed on tumor lesions in carcinoma samples, but not in adenoma (Fig. 5). In contrast, such strong activity was exhibited in both carcinoma and adenoma in rat samples (Fig. 6). The gelatinolytic activity was weakened by using FIZ-GI films in both species (Figs. $5,6)$.

\section{DISCUSSION}

In canine mammary tumors, activities of active forms of MMP-2 and MMP-9 have been reported to correlate with malignancy of tumors $[13,28]$. The results of the present study were consistent with the previous observation. Gelatinolytic activity of canine mammary tumors detected by FIZ was stronger in carcinoma samples than in adenoma, supporting the results of zymography. Such in situ gelatinolytic activity was significantly inhibited in the experiment using gelatin films including an MMP inhibitor (FIZ-GI film), indicating that the majority of the gelatinolytic activity may be due to MMPs activity. However, other proteases such as trypsin may be involved in the activity, because trypsin is 
known to be produced in several kinds of human cancer tissues $[4,26]$. Reverse zymography conducted in the present study showed that the activities of TIMP-1, TIMP-2, and TIMP-3 in canine mammary tumor samples were quite low, but significantly correlated with the malignancy of the tumors.

In cases of rat mammary tumors, the activity of only latent form of MMP-2 was higher in tumor tissues than in normal mammary tissues, but the activities of active MMP2 was at the same level in both tumors and normal tissue. Activities of latent or active forms of MMP-9 were undetectable in the present study. The present rat mammary tumors did not show any invasive and/or metastatic lesions (data not shown). The lack of invasion and metastasis in this animal model may be due to lower levels of activities of active MMP-2 and to the lack of MMP-9 activity. In addition, the higher activity of TIMPs may explain the characters of the rat tumor. In the present study, diffuse in situ gelatinolytic activities were detected in both malignant and benign rat tumor cases, in spite of low MMPs activities, indicating that other gelatinolytic enzymes might play a role. TIMPs are known to have an activity to promote cell growth [3, 14, 17, 27], as well as to inhibit MMPs activity. Therefore, higher activities of TIMPs detected in the rat malignant mammary tumors may contribute to both MMP inhibition and cell growth.

In the present study, the difference of MMPs activities between malignant and benign chemically induced rat mammary tumors was extremely less than those between malignant and benign canine mammary tumors. However, the difference of TIMPs activities was larger in rat cases than in canine cases. These results may indicate that the canine spontaneous malignant mammary tumors possess more aggressive nature such as metastatic and invasive behaviors than the rat chemically induced counterpart, resulting from the high level of MMPs and low level of TIMPs activities as well.

\section{REFERENCES}

1. Brodey, R. S., Goldschmidt, M. H. and Roszl, J. R. 1983. Canine mammary gland neoplasms. J. Am. Anim. Hosp. Assoc. 19: 61-90.

2. Garbett, E. A., Reed, M. W. and Brown, N. J. 1999. Proteolysis in human breast and colorectal cancer. Br. J. Cancer 81: 287293.

3. Hayakawa, T., Yamashita, K., Tanzawa, K., Uchijima, E. and Iwata, K. 1992. Growth-promoting activity of tissue inhibitor of metalloproteinases-1 (TIMP-1) for a wide range of cells. A possible new growth factor in serum. FEBS Lett. 298: 29-32.

4. Hirahara, F., Miyagi, Y., Miyagi, E., Yasumitsu, H., Koshikawa, N., Nagashima, Y., Kitamura, H., Minaguchi, H., Umeda, M. and Miyazaki, K. 1995. Trypsinogen expression in human ovarian carcinomas. Int. J. Cancer 63: 176-181.

5. Huggins, C., Grand, L. C. and Brillantes, F. P. 1961. Mammary cancer induced by a single feeding of polynuclear hydrocarbons, and its suppression. Nature (Lond.) 189: 204-207.

6. Iwata, H., Kobayashi, S., Iwase, H., Masaoka, A., Fujimoto, N. and Okada, Y. 1996. Production of matrix metalloproteinases and tissue inhibitors of metalloproteinases in human breast carcinomas. Jpn. J. Cancer Res. 87: 602-611.

7. Iwata, H., Yamamoto, M., Nemori, R., Mizutani, M., Iwase, T., Miura, S., Obata, Y., Hara, Y., Omoto, Y., Toyama, T., Yamashita, H., Iwase, H. and Kobayashi, S. 2001. Localization of gelatinolytic activity can be detected in breast cancer tissues by film in situ zymography. Breast Cancer 8: 111-115.

8. Kawamura, K., Kamiya, N., Suyama, T., Shimbo, M., Oosumi, N., Suzuki, H., Ueda, T., Tobe, T., Igarashi, T., Ito, H. and Ishikura, H. 2004. In situ gelatinolytic activity correlates with tumor progression and prognosis in patients with bladder cancer. J. Urol. 172: 1480-1484.

9. Kleiner, D. E. and Stetler-Stevenson, W. G. 1994. Quantitative zymography: detection of picogram quantities of gelatinases. Ann. Biochem. 218: 325-329.

10. Lambert, E., Dasse, E., Haye, B. and Petitfrere, E. 2004. TIMPs as multifacial proteins. Crit. Rev. Oncol. Hematol. 49: 187-198.

11. Liotta, L. A., Tryggvason, K., Garbisa, S., Hart, I., Foltz, C. M. and Shafie, S. 1980. Metastatic potential correlates with enzymatic degradation of basement membrane collagen. Nature (Lond.) 284: 67-68.

12. Liotta, L. A., Stetler-Stevenson, W. G. and Steeg, P. S. 1991. Cancer invasion and metastasis: positive and negative regulatory elements. Cancer Invest. 9: 543-551.

13. Loukopoulos, P., Mungall, B. A., Straw, R. C., Thornton, J. R. and Robinson, W. F. 2003. Matrix metalloproteinase-2 and -9 involvement in canine tumors. Vet. Pathol. 40: 382-394.

14. Luparello, C., Avanzato, G., Carella, C. and Pucci-Minafra, I. 1999. Tissue inhibitor of metalloprotease (TIMP)-1 and proliferative behaviour of clonal breast cancer cells. Breast Cancer Res. Treat. 54: 235-244.

15. Matrisian, L. M. 1990. Metalloproteinases and their inhibitors in matrix remodeling. Trends Genet. 6: 121-125.

16. Misdorp, W., Else, R. W., Hellemen, E. and Lipscomb, T. P. 1999. Histological Classification of Mammary Tumors of the Dog and the Cat, World Health Organization International Histological Classification of Tumors of Domestic Animals, Second Series Volume VII, AFIP, Washington, D.C.

17. Nemeth, J. A., Rafe, A., Steiner, M. and Goolsby, C. L. 1996. TIMP-2 growth-stimulatory activity: a concentration- and cell type-specific response in the presence of insulin. Exp. Cell Res. 224: $110-115$.

18. Oliver, G. W., Leferson, J. D., Stetler-Stevenson, W. G. and Kleiner, D. E. 1997. Quantitative reverse zymography: analysis of picogram amounts of metalloproteinase inhibitors using gelatinase A and B reverse zymograms. Ann. Biochem. 244: 161-166.

19. Papparella, S., Restucci, B., Paciello, O. and Maiolino, P. 2002. Expression of matrix metalloprotease-2 (MMP-2) and the activator membrane type 1 (MT1-MMP) in canine mammary carcinomas. J. Comp. Pathol. 126: 271-276.

20. Rha, S. Y., Kim, J. H., Roh, J. K., Lee, K. S., Min, J. S., Kim, B. S. and Chung, H. C. 1997. Sequential production and activation of matrix-metalloproteinase-9 (MMP-9) with breast cancer progression. Breast Cancer Res. Treat. 43: 175-181.

21. Rha, S. Y., Yang, W. I., Kim, J. H., Roh, J. K., Min, J. S., Lee, K. S., Kim, B. S. and Chung, H. C. 1998. Different expression patterns of MMP-2 and MMP-9 in breast cancer. Oncol. Rep. 5: $875-879$.

22. Russo, J., Gusterson, B. A., Rogers, A. E., Russo, I. H., Wellings, S. R. and van Zwieten, M. J. 1990. Comparative study of human and rat mammary tumorigenesis. Lab. Invest. 62: 244- 
278.

23. Russo, J. and Russo, I. H. 2000. Atlas and histologic classification of tumors of the rat mammary gland. J. Mammary Gland Biol. Neoplasia 5: 187-200.

24. Takano, S., Tsuboi, K., Matsumura, A., Sato, H. and Nose, T. 2001. Localization of gelatinase activities in glioma tissues by film in situ zymography. Brain Tumor Pathol. 18: 145-150.

25. Woodhouse, E. C., Chuaqui, R. F. and Liotta, L. A. 1997. General mechanisms of metastasis. Cancer 80: 1529-1537.

26. Yamamoto, H., Iku, S., Itoh, F., Tang, X., Hosokawa, M. and Imai, K. 2001. Association of trypsin expression with recurrence and poor prognosis in human esophageal squamous cell carcinoma. Cancer 91: 1324-1331.

27. Yang, T. T. and Hawkes, S. P. 1992. Role of the 21-kDa protein TIMP-3 in oncogenic transformation of cultured chicken embryo fibroblasts. Proc. Natl. Acad. Sci. U. S. A. 89: 10676 10680.

28. Yokota, H., Kumata, T., Taketaba, S., Kobayashi, T., Moue, H., Taniyama, H., Hirayama, K., Kagawa, Y., Itoh, N., Fujita, O., Nakade, T. and Yuasa, A. 2001. High expression of $92 \mathrm{kDa}$ type IV collagenase (matrix metalloproteinase-9) in canine mammary adenocarcinoma. Biochim. Biophys. Acta 1568: 712.

29. Zheng, K., Nagai, Y., Kishimoto, T., Yamazawa, K., Tate, S., Nemori, R., Hirai, Y., Sekiya, S. and Ishikura, H. 2002. A quantitative evaluation of active gelatinolytic sites in uterine endometrioid adenocarcinoma using film in situ zymography: association of stronger gelatinolysis with myometrial invasion. Jpn. J. Cancer Res. 93: 516-522. 